\title{
Analysis of the Direct-Current Bolometer Bridge
}

\author{
By David M. Kerns
}

\begin{abstract}
The behavior of a direct-current Wheatstone bridge with a nonlinear element (bolometer) in one arm, as used in the measurement of microwave power, is analyzed. Results of rather general applicability are obtained by ascribing arbitrary values to the resistances making up the bridge-network and by employing a resistance law of a general form for the bolometer element. Emphasis is placed upon the development of first-order theory in convenient form. General characteristics of the behavior of the type of network (and nonlinear element) under consideration are indicated. Results obtained include expression for the derivatives of galvanometer current with respect to radio-frequency power, ambient temperature, sourcevoltage, and source-resistance.
\end{abstract}

\section{Introduction}

Bolometers, consisting, for example, of bead thermistors or of fine platinum wires, have received wide use as microwave power measuring elements. In this application a bolometer is mounted in a wave-guide structure whose function is to enable the bolometer to absorb radio-frequency power from a source whose output is to be measured. The bolometer is also connected into a directcurrent (or low-frequency alternating-current) network whose functions are to detect or measure changes in bolometer resistance and to supply bias power to the bolometer. Thus in this arrangement the total electrical power dissipated in the bolometer consists of bias power plus any radiofrequency power that may be present. An essential property of the bolometer is, of course, that its resistance, through the dependence of the resistance upon temperature, is determined in a given environment by the total electrical power dissipated in the bolometer. Thus radio-frequency power may be measured in terms of the change of resistance produced by the application of the power to be measured, or it may be measured by the change in bias power required to keep the resistance constant. These principles have been employed in various, more or less elaborate, arrangements; ${ }^{1}$ technical details, however, are not needed for the purpose of this paper.

1 See, for example, Technique of microwave measurements 11, chapter 3 , MIT Radiation Laboratory series (McGraw-Hill Book Co., New York, N. Y., 1947).
This paper is concerned with the analysis of the behavior of what is probably the simplest bolometer circuit-arrangement that is useful in power measurements by the methods outlined. This arrangement is the Wheatstone-bridge network shown in figure 1 . The analysis of this network, however, is general: arbitrary values are ascribed to the resistances making up the network, and a resistance law of a general form is employed for the bolometer. Previously published analytical work on networks of the type considered (see footnote 1) is less general and in some respects less complete than that given here.

The point of view of this discussion, and the particular results derived, are perhaps especially appropriate in the planning and the analysis of precise experiments or measurements. Nevertheless, the treatment at the same time provides a basis for an understanding of some of the general characteristics of the behavior of the type of bolometer-bridge arrangement considered.

Although Wheatstone-bridge calculations can become tedious, it is found possible to put the calculations in a relatively convenient form and to obtain results in forms suited to numerical calculation. The notation and the methods used can be applied to the investigation of features of more specialized interest than those considered here. (A few identities that have been found of value in such investigations are given in the appendix.) 


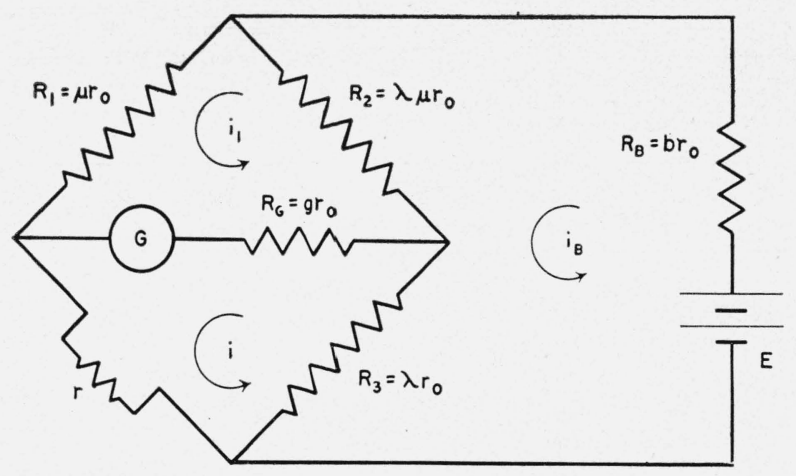

Figure 1. Network and notation.

\section{Expressions for the Currents}

The first task is to obtain convenient expressions for the currents in a Wheatstone-bridge network in which a bolometer of resistance $r$ constitutes one arm. The bolometer is a nonlinear element; $r$ is its direct-current resistance, defined by the equation $e=r i$, where $i$ is the bolometer current, and $e$ is the voltage across the bolometer terminals. In obtaining these first formulas, however, the value of $r$ is assumed to be given, so that the nonlinear behavior of the bolometer does not at first come into consideration.

The network and the notation are illustrated in figure 1. $R_{1}, R_{2}, R_{3}$ are the respective resistances of three arms of the bridge, $R_{G}$ is the resistance of the galvanometer branch, $R_{B}$ is the resistance of the battery branch, and $E$ is the battery voltage. Instead of employing these quantities directly as parameters of the network, it is of some convenience in numerical calculation and in the algebraic work to employ another set defined as follows. The bolometer resistance $r_{0}$ for which the bridge is balanced is given by

$$
r_{0} R_{2}=R_{1} R_{3} .
$$

It is convenient to introduce $r_{0}$ as an explicit parameter and to express $R_{1}, R_{2}, R_{3}$ in terms of $r_{0}$ and two ratios $\lambda, \mu$ by means of the equations

$$
R_{1}=\mu r_{0}, R_{2}=\lambda \mu r_{0}, R_{3}=\lambda r_{0} .
$$

Clearly these equations are such that (1) is automatically satisfied. The quantities $R_{B}, R_{G}$ will also be expressed in terms of $r_{0}$ by letting

$$
\left.\begin{array}{l}
R_{B}=b r_{0} \\
R_{G}=g r_{0}
\end{array}\right\}
$$

where $b, g$ are dimensionless parameters. The six quantities $r_{0}, \lambda, \mu, b, g, E$ will be regarded as the basic parameters of the network.

The system of equations for the mesh currents $i_{B}, i_{1}, i$ (which are illustrated in fig. 1) may be written

$$
\left.\begin{array}{rl}
E / r_{0} & =(b+\lambda+\lambda \mu) i_{B}-\lambda \mu i_{1}-\lambda i, \\
0 & =-\lambda \mu i_{B}+(g+\mu+\mu \lambda) i_{1}-g i, \\
0 & =-\lambda i_{B}-g i_{1}+\left(g+\lambda+r / r_{0}\right) i .
\end{array}\right\}
$$

The determinant of this system is

$$
D=\left|\begin{array}{lll}
b+\lambda+\lambda \mu & -\lambda \mu & -\lambda \\
-\lambda \mu & g+\mu+\mu \lambda & -g \\
-\lambda & -g & g+\lambda+r / r_{0}
\end{array}\right|
$$

Let $M_{B}, M_{1}, M$ denote respectively the cofactors of the first, the second, and the third elements in the first row of (5). One may then write for the mesh currents and the galvanometer current

$$
\begin{aligned}
i_{B}= & M_{B} E /\left(r_{0} D\right)=\left[(1+\lambda) g \mu+\left(r / r_{0}+\lambda\right)\right. \\
& (g+\mu+\mu \lambda)] E /\left(r_{0} D\right), \\
i_{1}= & M_{1} E /\left(r_{0} D\right)=\left(g+g \mu+\mu \lambda+\mu r / r_{0}\right) \lambda E /\left(r_{0} D\right), \\
i= & M E /\left(r_{0} D\right)=(g+g \mu+\mu \lambda+\mu) \lambda E /\left(r_{0} D\right), \\
i_{G}= & i-i_{1}=\left(1-r / r_{0}\right) \lambda \mu E /\left(r_{0} D\right) .
\end{aligned}
$$

When $r=r_{0}$ - that is, when the bridge is balancedthe cofactors just defined are evidently related by

$$
M=\left(M_{1}\right)_{0}=\left(M_{B}\right)_{0} \lambda /(1+\lambda),
$$

so that

$$
i_{0}=\left(i_{1}\right)_{0}=\left(i_{B}\right)_{0} \lambda /(1+\lambda),
$$

where, by inspection of the network,

$$
\left(i_{B}\right)_{0}=\left(E / r_{0}\right)\left[b+\lambda \frac{1+\mu}{1+\lambda}\right]^{-1} .
$$

(The subscript zero will be used generally to denote quantities evaluated for $r=r_{0}$.)

It is advantageous to write the determinant $D$ in the form

$$
D=p+q r / r_{0},
$$

where $p$ and $q$ do not depend upon $r$. By comparing (12) and (6) it is easily found that 


$$
D_{0}=(g+g \mu+\mu+\mu \lambda)(b+b \lambda+\lambda+\lambda \mu) .
$$

To evaluate $D$ for general values of $r$, both $p$ and $q$ must be known. The quantity $q$ is the coefficient of $r / r_{0}$ in the expansion of the determinant (5):

$$
q=(b+\lambda+\lambda \mu)(g+\mu+\mu \lambda)-(\lambda \mu)^{2} .
$$

An expression for $p$ is perhaps most easily obtained by noting that $D_{0}=p+q$ and calculating $p=D_{0}-q$. The result is

$$
p=(g+g \mu+\mu \lambda)(b+b \lambda+\lambda \mu)-g b .
$$

This expression may be verified, if desired, by expanding the determinant (5) with $r=0$, since $D$ evaluated for $r=\mathrm{o}$ is precisely $p$.

By Thévenin's theorem, the network into which the bolometer is connected may be represented by a suitable voltage $E^{\prime}$ and resistance $R^{\prime}$ in series. These quantities may be evaluated in a convenient form by using (13) and (8) to write

$$
i=\frac{E M / q}{r_{0} p / q+r}
$$

But $i$ is to be given by $E^{\prime} /\left(R^{\prime}+r\right)$; hence

$$
E^{\prime}=E M / q, \quad R^{\prime}=r_{0} p / q .
$$

Since $M, p, q$, are independent of $r$, the quantities $E^{\prime}, R^{\prime}$ are also independent of $r$, as indeed they must be.

The expression (9) for galvanometer current may now be put in a useful form, which shows explicitly the dependence upon $r$, viz,

$$
i_{G}=I \frac{r_{0}-r}{R^{\prime}+r},
$$

where $I$ is an abbreviation for $E \lambda \mu /\left(r_{0} q\right)$. As the final preliminary formula, the solution of this last equation for $r$ is recorded:

$$
r=r_{0}-\left(R^{\prime}+r_{0}\right) \frac{i_{G} / I}{1+i_{G} / I} .
$$

\section{Introduction of a General Bolometer Law}

The currents calculated in the preceding section are those that are obtained when the bolometer has the resistance $r$. To approach the essential problem of determining the bolometer resistance actually assumed under various operating conditions, some expression for the law of resistance of the bolometer must be introduced. It is both convenient and informative to employ a resistance law of a general form,

$$
r=r(T, P)
$$

where $r$ is the direct-current resistance of the bolometer, $T$ is the ambient temperature, and $P$ is the electrical power dissipated in the bolometer. This electrical power is considered to be the sum of two terms,

$$
P=P_{d c}+P_{r f},
$$

where $P_{d c}$ and $P_{r f}$ are respectively the directcurrent power and the radio-frequency power dissipated in the bolometer. Equations 20 and 21 contain the assumption that direct-current power and radio-frequency power have precisely equivalent effects on the bolometer resistance. Thermal lag prevents the bolometer resistance from following rapid changes of either ambient temperature or electrical power; the law (20) is therefore to be understood as giving equilibrium values of $r$. It may be noted that, in particular, the respective resistance laws used for bead-thermistor and platinum-wire bolometers can be written in the form (20).

The behavior of a bolometer in the neighborhood of a given operating point $(T, P)$ is characterized by two partial derivatives evaluated at that point. The basic equation is simply

$$
d r=\left(\frac{\partial r}{\partial T}\right)_{P} d T+\left(\frac{\partial r}{\partial P}\right)_{T} d P .
$$

If $r$ is held constant, the resistance law $r=r(T, P)$ defines a relationship between $T$ and $P$. From the rule for the differentiation of implicit functions (or, indeed, directly from the differential equation 22 ), one may write

$$
\left(\frac{\partial P}{\partial T}\right)_{r}=-\left(\frac{\partial r}{\partial T}\right)_{P} /\left(\frac{\partial r}{\partial P}\right)_{T}
$$

Any two of the derivatives in this equation determine the third. In practice it is sometimes convenient to make use of this fact, determining $(\partial r / \partial T)_{P}$ by measuring the other two derivatives rather than by measuring it directly. 
Upon introducing the notation

$$
\left(\frac{\partial r}{\partial P}\right)_{T}=\gamma,\left(\frac{\partial P}{\partial T}\right)_{r}=-\frac{1}{c}
$$

the identity (23) may be written

$$
\left(\frac{\partial r}{\partial T}\right)_{P}=\frac{\gamma}{c}
$$

and the expression (22) for $d r$ becomes

$$
d r=\gamma(d T / c+d P) .
$$

The important parameter $\gamma$, which is the rate of change of bolometer resistance with respect to electrical power at a given ambient temperature, is the "ohms-per-watt" coefficient characteristic of the bolometer. The differential coefficient $(\partial P / \partial T)_{r}$, as suggested by the notation chosen for it, is in many cases a constant to a good approximation. But it should be remembered that both $\gamma$ and $c$ are, in general, functions of $T$ and $P$.

The resistance law (20) may be written in the form

$$
P=P(T, r),
$$

which represents (20) solved for $P$. For $r=r_{0}$, $P_{r f}=0$, and any fixed value of $T$, the bolometer current is $i_{0}$, where

$$
r_{0} i_{0}^{2}=P\left(T, r_{0}\right),
$$

since $P=P_{d c}=r_{0} i_{0}^{2}$. The current $i_{0}$ is also given by

$$
i_{0}=E^{\prime} /\left(R^{\prime}+r_{0}\right),
$$

where $E^{\prime}, R^{\prime}$, as defined in section II, represent the bridge into which the bolometer is connected. By eliminating $i_{0}$ between the last two equations, one obtains

$$
E^{\prime} /\left(E^{\prime}+r_{0}\right)=\sqrt{P\left(T, r_{0}\right) / r_{0}} .
$$

The process of balancing the bridge consists of adjusting the bridge-parameters involved so that (27) is in fact satisfied. The left-hand side of (27) may be written in a form that exhibits the parameters involved:

$$
E^{\prime} /\left(R^{\prime}+r_{0}\right)=(\lambda E)\left[r_{0}(b+b \lambda+\lambda+\lambda \mu)\right]^{-1} .
$$

In many cases $\lambda$ and $\mu$, as well as $r_{0}$, are preferably not varied; in these cases, $b$ or $E$ (or both) may be adjusted to obtain bridge-balance. The bolometer current $i_{0}$ obtained when the bridge is balanced (with $P_{r f}=0$ ) is often called the bias current.

The fundamental equations applying to the socalled balanced-bridge method of measuring radiofrequency power are very easily obtained. If the bridge is balanced with radio-frequency power present, the corresponding bolometer current satisfies

$$
r_{0} i^{2}=P\left(T, r_{0}\right)-P_{r f} .
$$

On the other hand, (26) holds when the bridge is balanced with no radio-frequency power present. Equations 26 and 28 combined yield the equation

$$
P_{r f}=r_{0}\left(\dot{i}_{0}^{2}-i^{2}\right),
$$

for the determination of $P_{r f}$ in terms of $r_{0}$ and measured values of $i$ and $i_{0}$. Granted the assumption of the form of the bolometer law itself, there remain two basic assumptions involved in (29). These are discussed briefly in the following two paragraphs.

Equation 29 involves the assumption that $i_{0}$ and $i$ are measured under conditions of thermal equilibrium at the same ambient temperature $T$. If $i_{0}$ is measured at temperature $T$ and $i$ at temperature $T+d T$, eq 29 is replaced by the approximate equation

$$
\left.\begin{array}{rl}
P_{r f} & =r_{0}\left(i_{0}^{2}-i^{2}\right)+\left(\frac{\partial P}{\partial T}\right)_{r} d T \\
& =r_{0}\left(i_{0}^{2}-i^{2}\right)-d T / c .
\end{array}\right\}
$$

This equation is correct to the first order, provided that the somewhat unrealistic condition of thermal equilibrium at each of the two temperatures $T$ and $T+d T$ is fulfilled.

The value of $r_{0}$, as a parameter of the bridge, can be made very precise. But the use of $r=r_{0}$ in 29 implies that the bridge is exactly balanced when the two currents are measured. The uncertainty in obtaining $r=r_{0}$ is determined largely by the useful sensitivity of the bridge with respect to unbalance. It is convenient to estimate the corresponding contribution to the uncertainty in $P_{r f}$ directly (rather than by first finding the uncertainty in $r$ ) by means of an equation of the form

$$
d P_{\tau f}=\frac{\partial P_{r f}}{\partial i_{G}} d i_{G}
$$


with $d i_{G}$ placed equal to the smallest detectable change in galvanometer current. The quantity $\left(\partial i_{G} / \partial P_{r f}\right)$, which is frequently called the " $r f$ sensitivity," can be calculated by means of formulas to be derived later.

In the so-called unbalanced-bridge, or deflection method of measuring radio-frequency power, no adjustment of the initially balanced bridge is made when the radio-frequency power is applied, and the resulting value of galvanometer current is taken as a measure of the power. In this method the nonlinearity of the relationship of $P_{r f}$ to $i_{G}$ is naturally of some concern. For this reason an extension of 31 to include the quadratic term in $i_{G}$ is given (section $\mathrm{V}$ ).

In the treatment of nonlinear circuit elements, the nonlinear element is often described by a law written in the form

$$
e=f(i)
$$

Such a relationship - or rather a family of such relationships, with $P_{r f}=$ constant, $T=$ constant as parameters - is indeed defined by the bolometer resistance law in the form already adopted.

If an element having the $e-i$ characteristic (32) is connected to a source consisting of a voltage $E^{\prime}$ and a resistance $R^{\prime}$ in series, the resulting current must simultaneously satisfy the two equations

$$
\begin{aligned}
& e=f(i), \\
& e=E^{\prime}-i R^{\prime}
\end{aligned}
$$

In other words, $i$ must satisfy the equation

$$
f(i)=E^{\prime}-i R^{\prime}
$$

Let $E^{\prime}, R^{\prime}$ receive increments $d E^{\prime}, d R^{\prime}$, respectively. Then the corresponding differential of $i$ is given by

$$
d i=\frac{d E^{\prime}-i d R^{\prime}}{R^{\prime}+r_{v}}
$$

where $r_{v}$, denoting the derivative of $f$ with respect to $i$, is the variational resistance (for slow variations) of the element. Thus with respect to small changes in $E^{\prime}$ and $R^{\prime}$, the nonlinear element behaves like an ohmic element of resistance $r_{v}$.

An expression for $r_{v}$ may be derived from the resistance law in the original form (20). The equation

\section{Bolometer-Bridge Analysis}

$$
e-i r\left(T, P_{r r}+e i\right)=0,
$$

which is obtained from (20) by substituting $r=e / i$ and $P_{d c}=e i$ in that equation, may be regarded as an identity in $e$ or $i$ for fixed values of $T$ and $P_{r f}$, and thereby defines $e=f(i)$. The variational resistance is obtained from (35) as the derivative of an implicit function:

$$
r_{v}=-\frac{\frac{\partial}{\partial i}\left[e-i r\left(T, P_{r f}+e i\right]\right.}{\frac{\partial}{\partial e}\left[e-i r\left(T, P_{r f}+e i\right]\right.}=-\frac{-r-i e \gamma}{1-i^{2} \gamma},
$$

where $\gamma=(\partial r / \partial P)_{T}$ as previously defined. In a slightly modified form

$$
r_{o}=r \frac{1+i^{2} \gamma}{1-i^{2} \gamma}
$$

This identity will be used to obtain an alternative form of one of the expressions to be derived in the next section.

\section{First-Order Theory}

Since, so far as the bolometer is concerned, the bridge is completely represented by the equivalent $E^{\prime}, R^{\prime}$, the effects upon the bolometer of changes in any of the basic parameters characterizing the bridge in detail may be evaluated in terms of corresponding changes in $E^{\prime}$ and $R^{\prime}$. Accordingly, in setting up the basic equation for a complete first-order theory, the quantities $E^{\prime}, R^{\prime}, r, T, P_{r s}$ are selected as variables.

Equation $22^{\prime}$ may be written

$$
d r=\gamma\left(d T / c+d P_{r f}+d P_{d c}\right),
$$

where $d P$ is separated into its parts $d P_{r f}, d P_{d c}$. Since $P_{d c}$ is given in terms of $E^{\prime}, R^{\prime}, r$ by $P_{d c}=$ $r E^{\prime 2} /\left(R^{\prime}+r\right)^{2}$, the differential of $P_{d c}$ is given by

$$
d P_{d c}=\frac{2 E^{\prime} r d E^{\prime}}{\left(R^{\prime}+r\right)^{2}}+\frac{E^{\prime 2}\left(R^{\prime}-r\right) d r}{\left(R^{\prime}+r\right)^{3}}-\frac{2 E^{\prime 2} r d R^{\prime}}{\left(R^{\prime}+r\right)^{3}},
$$

which may be more conveniently written as

$$
d P_{d c}=\eta i^{2} d r+2 P_{d c}\left(d E^{\prime}-i d R^{\prime}\right) / E^{\prime},
$$

where, in addition to the obvious relation $i=$ $E^{\prime} /\left(R^{\prime}+r\right)$, the notation

$$
\eta=\frac{R^{\prime}-r}{R^{\prime}+r}
$$


has been used. If this expression for $d P_{d c}$ is substituted into the above expression for $d r$, one obtains upon solving for $d r$,

$$
d r=\gamma_{e}\left[d T / c+d P_{r f}+2 P_{d c}\left(d E^{\prime}-i d R^{\prime}\right) / E^{\prime}\right],
$$

where

$$
\gamma_{e}=\frac{\gamma}{1-i^{2} \gamma \eta}
$$

Equation 37 serves as the basic equation for the first-order theory.

The role of the factor $\gamma_{e}$ in (37) is significant. If $E^{\prime}, R^{\prime}, T$ are held constant, equation 37 states that $d r=\gamma_{e} d P_{r f}$. Thus $\gamma_{e}$ might be denoted by $\left(\partial r / \partial P_{r f}\right)_{T}$ and is an effective ohms-per-watt coefficient, which depends not only upon the characteristics of the bolometer but also upon the network into which the bolometer is connected. $\gamma_{e}$, as a multipler in (37), includes the effect of the change in $P_{d c}$ concomitant to a change in any one of the variables $T, P_{r f}, E^{\prime}, R^{\prime}$. The quantity $\eta$ (which appears in the denominator of the expression (38) for $\gamma_{e}$ ) is in fact of the form of a reflection coefficient and is a measure of the mismatch between the equivalent source and its load.

Under certain conditions very large values for $\gamma_{e}$ can be obtained. By inspection of the denominator of (38) it is apparent that $\left|\gamma_{e}\right| \rightarrow \infty$ if

$$
\gamma \rightarrow \frac{1}{i^{2} \eta}=\frac{1}{i^{2}} \cdot \frac{R^{\prime}+r}{R^{\prime}-r}
$$

Comparing this expression with the relation (36), it is evident that this condition is equivalent to $r_{v} \rightarrow-R^{\prime}$, as indeed might be expected. This fact is also apparent in another form for the expression for $\gamma_{e}$, which is obtained by using (36) to eliminate $\gamma$ from (38). The result is

$$
\gamma_{e}=\frac{R^{\prime}+r}{2 P_{d c}} \cdot \frac{r_{v}-r}{r_{v}+R^{\prime}} .
$$

Thus when $r_{v}$ is negative (as it is for a bead thermistor under typical operating conditions), arbitrarily large values of $\gamma_{e}$ can in principle be obtained by making $R^{\prime} \rightarrow-r_{v}$. Unfortunately this fact is of somewhat limited usefulness in power measurement, because, as is evident from (37), the sensitivity of $r$ to changes in all the other variables increases when the sensitivity to radiofrequency power - the desired sensitivity - is increased.
So far, this discussion has dealt essentially with changes produced in $r$. The sensitivity of the galvanometer current (which, rather than $r$, is the observed quantity) to changes in radio-frequency power, temperature, source-voltage $(E)$, and source-resistance $\left(R_{B}\right)$ will now be calculated. These sensitivities are perhaps most naturally denoted as partial derivatives, but neither the partial nor the total derivative notation is adequate to express their precise meanings. The selected independent variable, the dependent variables, and the quantities held constant must be noted in each case.

a. $R F$ power sensitivity, $S_{r f}$. Consider first the sensitivity of galvanometer current to changes of radio-frequency power, with the temperature and all network parameters (including $E$ ) being held constant. Under these conditions the firstorder change $d i_{G}$ in galvanometer current corresponding to a change $d r$ in bolometer resistance is given by

$$
d i_{G}=\frac{\partial}{\partial r}\left(I \frac{r_{0}-r}{R^{\prime}+r}\right) d r=-I \frac{R^{\prime}+r_{0}}{\left(R^{\prime}+r\right)^{2}} d r,
$$

where the expression (18) for $i_{G}$ is used. From (37) one finds

$$
d r=\gamma_{e} d P_{r f},
$$

since $T, E^{\prime}, R^{\prime}$ are here constants. Hence, letting $S_{r f}=\partial i_{G} / \partial P_{r f}$, one has the result

$$
S_{r f}=-I \gamma_{e} \frac{R^{\prime}+r_{0}}{\left(R^{\prime}+r\right)^{2}} .
$$

b. Temperature sensitivity, $S_{T}$. The definition of the temperature sensitivity differs from that for $S_{r f}$ only in that $T$ instead of $P_{r f}$ is taken as the independent variable. Hence, from (37),

$$
d r=\gamma_{e} d T / c .
$$

Combining this with (40), and using the notation $S_{T}=\partial i_{G} / \partial T$, the result may be written

$$
S_{T}=S_{r f} / c .
$$

c. Sensitivity to source-voltage, $S_{E}$. The sensitivity of galvanometer current to changes in sourcevoltage $E$ is calculated by considering $E$ as the independent variable, all other circuit parameters, as well as $T$ and $P_{r f}$, being held constant. In this case (40) must be replaced by the more general expression 


$$
d i_{G}=\frac{i_{G}}{E} d E-I \frac{R^{\prime}+r_{0}}{\left(R^{\prime}+r\right)^{2}} d r,
$$

in which the additional term appears because (unless the bridge is initially balanced) $i_{G}$ depends upon $E$ directly, as well as indirectly through the dependence of $r$ on $E$. From (37),

$$
d r=2 \gamma_{e} P_{d c} d E^{\prime} / E^{\prime} .
$$

Since the network parameters are not varied, $d E^{\prime} / E^{\prime}$ is equal to $d E / E$. It follows that

$$
S_{E}=\frac{\partial i_{G}}{\partial E}=\frac{i_{G}}{E}-\frac{2 \gamma_{e} P_{d c} I\left(R^{\prime}+r_{0}\right)}{E\left(R^{\prime}+r\right)^{2}},
$$

where $S_{E}$ is the desired sensitivity.

d. Sensitivity to source-resistance, $S_{B}$. The sensitivity of galvanometer current to changes in source-resistance is obtained by considering $b$ as the independent variable, all other network parameters, as well as $T$ and $P_{r f}$, being held constant. From the expression (18) for $i_{G}$ it is apparent that the expression corresponding to (40), if written out, would have three terms, since $q, R^{\prime}$, and $r$ depend upon $b$. Moreover $d r$ is given by

$$
d r=2 \gamma_{e} P_{d c}\left(d E^{\prime}-i d R^{\prime}\right),
$$

wherein $d E^{\prime}$ and $d R^{\prime}$ are determined by the dependence of $E^{\prime}$ and $R^{\prime}$ upor $b$. (The dependence of $E^{\prime}$ and $R^{\prime}$ upon $b$ is given explicitly by eq 15 , 16, and 17. Although the calculations implied by these remarks can be carried out in fairly compact form, the result may be obtained by the following simpler method. If $E$ and $R_{B}$ are both varied, then $d i_{G}$ is given by

$$
d i_{G}=S_{E} d E+S_{B} d R_{B},
$$

where $S_{B}$ denotes the sensitivity to be calculated. Now the bridge as a whole may be considered as a nonlinear element connected to the source consisting of $E$ and $R_{B}$ in series, and the principle expressed by (34) may be applied to this circuit:

$$
d i_{B}=\frac{d E-i_{B} d R_{B}}{R_{B}+F^{\prime}\left(i_{B}\right)}
$$

In this expression $i_{B}$ is battery-branch current (as defined in section II), and $F^{\prime}\left(i_{B}\right)$ represents the variational resistance of the bridge as a whole. Suppose that $E$ and $R_{B}$ are varied in such a way that $d i_{G}=0$; this requires $d i_{B}=0$, which in turn requires that $d E=i_{B} d R_{B}$. Hence, from the above expression for $d i_{G}$,

$$
0=\left(S_{E} i_{B}+S_{B}\right) d R_{B},
$$

and the desired result is given by the simple relation $^{2}$

$$
S_{B}=-i_{B} S_{E}
$$

The expressions for the sensitivities calculated above simplify somewhat when evaluated for $r=r_{0}$. It may be observed that

$$
\left(S_{r f}\right)_{0}=\frac{E}{2 P_{d c}}\left(S_{E}\right)_{0}
$$

Also, by (44),

$$
\left(S_{r f}\right)_{0}=-\frac{E}{2 i_{B} P_{d c}}\left(S_{B}\right)_{0} .
$$

These equations suggest a method of evaluating $\left(\mathrm{S}_{r}\right)_{0}$ by means of direct-current measurements.

\section{A Second-Order Calculation}

Taking the resistance law in the form (25), separating $P$ into its components $P_{r f}, P_{d c}$, and replacing $P_{d c}$ by $E^{\prime 2} r /\left(R^{\prime}+r\right)^{2}$, one obtains

$$
P_{r f}=P(T, r)-E^{\prime 2} r /\left(R^{\prime}+r\right)^{2} .
$$

$P_{r f}$ becomes a function of $i_{G}$ through the dependence of $r$ upon $i_{G}$, as given by eq 19 ,

$$
r-r_{0}=-\left(R^{\prime}+r_{0}\right) \frac{i_{G} / I}{1+i_{G} / I} .
$$

Equation 45, with the implied elimination of $r$ by means of (19), represents an analytical expression for $P_{r f}\left(i_{G}\right)$, i. e., for the calibration curve of the bolometer bridge as used in the unbalanced bridge method of measuring radio-frequency power. For some purposes a power-series expansion of $P_{r f}\left(i_{G}\right)$ is useful.

Elimination of $r$ from the last term of (45) yields

$$
P_{d c}=i_{0}^{2}\left[r_{0}+\left(r_{0}-R^{\prime}\right) \frac{i_{G}}{I}-R^{\prime}\left(\frac{i_{G}}{I}\right)^{2}\right] .
$$

Thus $P_{d c}$ is given in general and without approximation as a quadratic in $i_{G}$. Let it be assumed that $P(T, r)$ can be expanded in the series

$$
\begin{aligned}
P(T, r)= & P\left(T, r_{0}\right)+P_{1}\left(T, r_{0}\right)\left(r-r_{0}\right)+\frac{1}{2} P_{2}\left(T, r_{0}\right)(r- \\
& \left.r_{0}\right)^{2}+\ldots,
\end{aligned}
$$

\footnotetext{
2 This result could have been obtained, with a slight gain in brevity, by employing a standard network theorem generally known as the compensation theorem. The argument given a voids any question of the applicability of the theorem to a circuit involving a nonlinear element.
} 
convergent for $\left|\mathrm{r}-r_{0}\right|<\rho$, say, where $P_{1}, P_{2} \ldots$ denote the successive derivatives of $P(T, r)$ with respect to $r$. The expression for $r-r_{0}$ can be expanded in the series

$$
r-r_{0}=\left(R^{\prime}+r_{0}\right)\left[-\frac{i_{G}}{I}+\left(\frac{i_{G}}{I}\right)^{2}-\left(\frac{i_{G}}{I}\right)^{3}+\ldots\right],
$$

which converges for $\left|i_{G}\right|<I$. (The algebraically largest and smallest values of $i_{G}$ are $I$ and $-\left(R^{\prime} / r_{0}\right) I$, corresponding respectively to $r \rightarrow \infty$ and $r=0$. Thus only if $R^{\prime} \leqq r_{0}$ does the series for $r-r_{0}$ converge for all physically possible values of $i_{G}$.) Substituting this series into the series for $P(T, r)$, ordering according to powers of $i_{G}$, and dropping powers of $i_{G}$ higher than the second, one finds

$P(T, r)=P\left(T, r_{0}\right)-P_{1}\left(T, r_{0}\right)\left(R^{\prime}+r_{0}\right) \frac{i_{G}}{I}+$

$\left[\frac{1}{2} P_{2}\left(T, r_{0}\right)\left(R^{\prime}+r_{0}\right)^{2}+P_{1}\left(T, r_{0}\right)\left(R^{\prime}+r_{0}\right)\right]\left(\frac{i_{G}}{I}\right)^{2}+\cdot \cdot$

Here $i_{G}$ is understood to be restricted so that the conditions $\left|r-r_{0}\right|<\rho$ and $\left|i_{G}\right|<I$ are both satisfied. Equations 46 and 47 together yield the expansion of (45):

$P_{r f}=P\left(T, r_{0}\right)-r_{0} i_{0}^{2}-$

$\left[P_{1}\left(T, r_{0}\right)\left(R^{\prime}+r_{0}\right)+i_{0}^{2}\left(r_{0}-R^{\prime}\right)\right] \frac{i_{G}}{I}+$

$\left[\frac{1}{2} P_{2}\left(T, r_{0}\right)\left(R^{\prime}+r_{0}\right)^{2}+P_{1}\left(T, r_{0}\right)\left(R^{\prime}+r_{0}\right)+i_{0}^{2} R^{\prime}\right]\left(\frac{i_{G}}{I}\right)^{2}+\cdots$

If, as is assumed, $P_{r f}=0$ when $i_{G}=0$, then the constant term vanishes. Further, the coefficient of $i_{G}$ is necessarily equal to $\left(S_{r f}\right)^{-1}$ (to verify, note that $P_{1}(T, r)=1 / \gamma$, and compare with (41)). Hence the final second-order expression may be written

$P_{r f}=\left(S_{r f}\right)_{0}^{-1} i_{G}+$

$\left[\frac{1}{2} P_{2}\left(T, r_{0}\right)\left(R^{\prime}+r_{0}\right)^{2}+P_{1}\left(T, r_{0}\right)\left(R^{\prime}+r_{0}\right)+i_{0}^{2} R^{\prime}\right]\left(\frac{i_{G}}{I}\right)^{2}$.

\section{Appendix}

In this appendix are derived several identities that are useful in various extensions of the calculations of the text. These identities involve primarily the quantities $M, p, q$ derived from the determinant $D$, and the determinant $D$ itself. All of the quantities involved (except one as noted) are as defined in section II of the text. The relationships to be obtained hold for arbitrary values of the quantities $r, r_{0}, \lambda, \mu, b, g$ appearing in the expression (5) for $D$. These quantities here may be, and are, considered as independent variables.

The calculations are conveniently based upon a theorem on determinants ${ }^{3}$ that states that if $D$ is any determinant and $S$ is the second minor obtained from it by striking out its $i^{\text {th }}$ and $k^{\text {th }}$ rows and its $j^{\text {th }}$ and $l^{\text {th }}$ columns, and if $A_{i j}$ denotes the cofactor of the element that stands in the $i^{\text {th }}$ row and the $j^{\text {th }}$ column of $D$, then

$$
\left|\begin{array}{cc}
A_{i j} & A_{i l} \\
A_{k i} & A_{k l}
\end{array}\right|=(-1)^{i+i+k+l} D S .
$$

This theorem, applied to the determinant (5) with $i=j=1, k=l=3$, yields

$$
\left|\begin{array}{ll}
M_{B} & M \\
M & q
\end{array}\right|=(g+\mu+\mu \lambda) D .
$$

Now $g+\mu+\mu \lambda$ is equal to $\partial q / \partial b$, and $M_{B}$ is equal to $\partial D / \partial b$. Hence the above equation may be written

$$
M^{2}=q \frac{\partial D}{\partial b}-D \frac{\partial q}{\partial b} .
$$

This identity is a key to the establishment of others of more immediate physical significance. Thus, the derivative of the equivalent resistance $R^{\prime}$ with respect to batterybranch resistance $R_{B}$ is

$$
\frac{\partial}{\partial b}\left(\frac{p}{q}\right)=\frac{\partial}{\partial b}\left(\frac{D}{q}\right)=\left(q \frac{\partial D}{\partial b}-D \frac{\partial q}{\partial b}\right) / q^{2}
$$

Therefore, by (49),

$$
\frac{\partial}{\partial b}\left(\frac{p}{q}\right)=\left(\frac{M}{q}\right)^{2}
$$

The derivative of the quantity $\eta=\left(R^{\prime}-r\right) /\left(R^{\prime}+r\right)$ (introduced in connection with eq 37 ) with respect to the parameter $b$ is easily obtained:

$$
\frac{\partial \eta}{\partial b}=\frac{\partial \eta}{\partial R^{\prime}} \frac{\partial R^{\prime}}{\partial b}=\frac{2 r r_{0}}{\left(R^{\prime}+r\right)^{2}}\left(\frac{M}{q}\right)^{2} ;
$$

upon noting that $q\left(R^{\prime}+r\right)=r_{0}\left(p+q r / r_{0}\right)=r_{0} D$, one has

$$
\frac{\partial \eta}{\partial b}=2\left(\frac{r}{r_{0}}\right)\left(\frac{M}{D}\right)^{2} \text {. }
$$

There are corresponding expressions for the derivatives of $R^{\prime}$ and $\eta$ with respect to galvanometer resistance. These are readily obtained by making use of the fact that the interchanges

$$
\begin{array}{ll}
g \rightarrow b, & \lambda \rightarrow \mu, \\
b \rightarrow g, & \mu \rightarrow \lambda,
\end{array}
$$

3 M. Bôcher, Introduction to higher algebra, p. 33 (Macmillan Co., New York, N. Y., 1907). 
leave the quantities $p$ and $q$ unchanged. That is, if $(*)$ denotes the quantity as transformed by the set of interchanges, then

$$
q^{*}=q, p^{*}=p
$$

From this property and from eq 50 and 51 , it follows that

and that

$$
\frac{\partial}{\partial g}\left(\frac{p}{q}\right)=\left[\frac{\partial}{\partial b}\left(\frac{p}{q}\right)\right]^{*}=\frac{\left(M^{2}\right)^{*}}{q^{2}}
$$

$$
\left(\frac{\partial \eta}{\partial g}\right)=\left(\frac{\partial \eta}{\partial b}\right)^{*}=2\left(\frac{r}{r_{0}}\right) \frac{\left(M^{2}\right)^{*}}{D^{2}}
$$

These two results are put into final form by making use of the relation $M M^{*}=\lambda \mu D_{0}$, which is evident from the explicit formulas for $M$ and $D_{0}$. One obtains

$$
\begin{gathered}
\frac{\partial}{\partial g}\left(\frac{p}{q}\right)=\left(\frac{\lambda \mu D_{0}}{M q}\right)^{2} \\
\frac{\partial \eta}{\partial g}=2\left(\frac{r}{r_{0}}\right)\left(\frac{\lambda \mu D_{0}}{M D}\right)^{2} .
\end{gathered}
$$

Equations 51 and 53 simplify to some extent when, in particular, $r=r_{0}$. In some cases one might wish to make use of the fact that $M / \lambda$ is a factor of $D_{0}$.

The author is grateful to Harold Lyons, Chief of the Microwave Standards Section, National Bureau of Standards, for encouragement in the preparation of this paper.

Washington, October 6, 1948. 